\title{
The structured environments of embedded star-forming cores ${ }^{\star}$ PACS and SPIRE mapping of the enigmatic outflow source UYSO 1
}

\author{
H. Linz ${ }^{1}$, O. Krause ${ }^{1}$, H. Beuther ${ }^{1}$, Th. Henning ${ }^{1}$, R. Klein ${ }^{2}$, M. Nielbock ${ }^{1}$, B. Stecklum ${ }^{3}$, \\ J. Steinacker ${ }^{4,1}$, and A. Stutz ${ }^{1}$ \\ 1 Max-Planck-Instritut für Astronomie (MPIA) Heidelberg, Königstuhl 17, 69117 Heidelberg, Germany \\ e-mail: [linz;krause; beuther; henning;nielbock; stein; stutz] @mpia.de \\ 2 Space Sciences Laboratory, University of California, Berkeley, CA 94720, USA; e-mail: r_klein@calmail . berkeley.edu \\ 3 Thüringer Landessternwarte Tautenburg (TLS), Sternwarte 5, 07778 Tautenburg, Germany \\ e-mail: stecklum@tls-tautenburg.de \\ 4 LERMA, Observatoire de Paris, 61 Av. de l'Observatoire, 75014 Paris, France
}

Received 31 March 2010 / Accepted 3 May 2010

\section{ABSTRACT}

\begin{abstract}
The intermediate-mass star-forming core UYSO 1 has previously been found to exhibit intriguing features. While deeply embedded and previously only identified by means of its (sub-)millimeter emission, it drives two powerful, dynamically young, molecular outflows. Although the process of star formation has obviously started, the chemical composition is still pristine. We present Herschel PACS and SPIRE continuum data of this presumably very young region. The now complete coverage of the spectral energy peak allows us to precisely constrain the elevated temperature of $26-28 \mathrm{~K}$ for the main bulge of gas associated with UYSO1, which is located at the interface between the hot H II region Sh 2-297 and the cold dark nebula LDN 1657A. Furthermore, the data identify cooler compact far-infrared sources of just a few solar masses, hidden in this neighbouring dark cloud.
\end{abstract}

Key words. stars: formation - infrared: stars: - infrared: ISM - ISM: structure - stars: individual: NAME UYSO 1

\section{Introduction}

Star formation occurs predominantly in structured environments in the case of intermediate and high-mass star formation. In particular, one often finds different stages of evolution located within close proximity. This poses a challenge for working on the earliest phases of star formation, which can only be revealed with long-wavelength observations. To observe these deeply embedded objects at the spectral peak of their emission, airborne or satellite missions are necessary, which in the past delivered only a very modest spatial resolution $\left(>20^{\prime \prime}\right)$. We use ESA's new farIR and sub-millimeter satellite Herschel (Pilbratt et al. 2010) with its unprecedented spatial resolution - to scrutinise a sample of very young low- and high-mass star-forming cores within our programme "Early Phases of Star Formation" (EPoS, PI: O. Krause). One of these targets is a core located in the Canis Majoris OB1/R1 region (e.g., Gregorio-Hetem et al. 2009) of the outer Galaxy at a distance of $\sim 1 \mathrm{kpc}$. Originally detected as a distinct submillimetre source in the vicinity of IRAS 07029-1215 (Forbrich et al. 2004), it was coyly named "UYSO 1" (unidentified young stellar object 1) since it was only detected at 450 and $850 \mu \mathrm{m}$ and seemed to have no counterpart at other wavelengths.

In the optical, the entire region is dominated by the strong HII region Sh 2-297, excited by the early B star HD 53623. The mid-IR emission is dominated by warm dust and PAH emission from the associated southern PDR. All these sources, however,

* Herschel is an ESA space observatory, with its science instruments PACS, SPIRE, and HIFI provided by European-led Principal Investigator consortia, and with important participation from NASA. do not coincide with UYSO 1, located more than $1^{\prime}$ to the northwest. Further to the west, one finds the optical dark cloud LDN 1657A, about which little is known. Members of our group carried out a multiwavelength study of UYSO 1 (Forbrich et al. 2009), which revealed more puzzles. The source drives a dynamically young, but strong CO molecular outflow. The imprints of two crossed jets are visible in shocked $\mathrm{H}_{2}$ emission in the nearIR, and their vertex is very close to the position of UYSO 1. Millimeter continuum interferometry resolved the source into two peaks separated by 4 .'2. Spitzer/MIPS data indicated that at 24 and $70 \mu \mathrm{m}$, UYSO 1 is still too deeply embedded to be directly detected, a finding we revise in the present paper for $70 \mu \mathrm{m}$ with Herschel. In addition to the core being chemically pristine and showing no signs of more evolved chemistry, this object is very interesting for the investigation of the early evolution of (intermediate-mass) protostars.

\section{Observations and data reduction}

The object UYSO 1 and its vicinity were observed in the blue (70 $\mu \mathrm{m})$, green $(100 \mu \mathrm{m})$, and red $(160 \mu \mathrm{m})$ filters with the PACS instrument (Poglitsch et al. 2010) aboard Herschel on October 9 , 2009, within the science demonstration program. Scan maps in two orthogonal directions with scan leg lengths of $7^{\prime}$ were obtained with a scan speed of $20^{\prime \prime} / \mathrm{s}$, for which the spatial resolution is $5{ }^{\prime \prime} 6,66^{\prime \prime} 8$, and $11^{\prime \prime} .3$, respectively. The raw data were reduced with the HIPE software (e.g., Poglitsch et al. 2010), version 3.0, build 455 . Beside the standard steps leading to level-1 calibrated data, a second-level deglitching and a correction for offsets in 


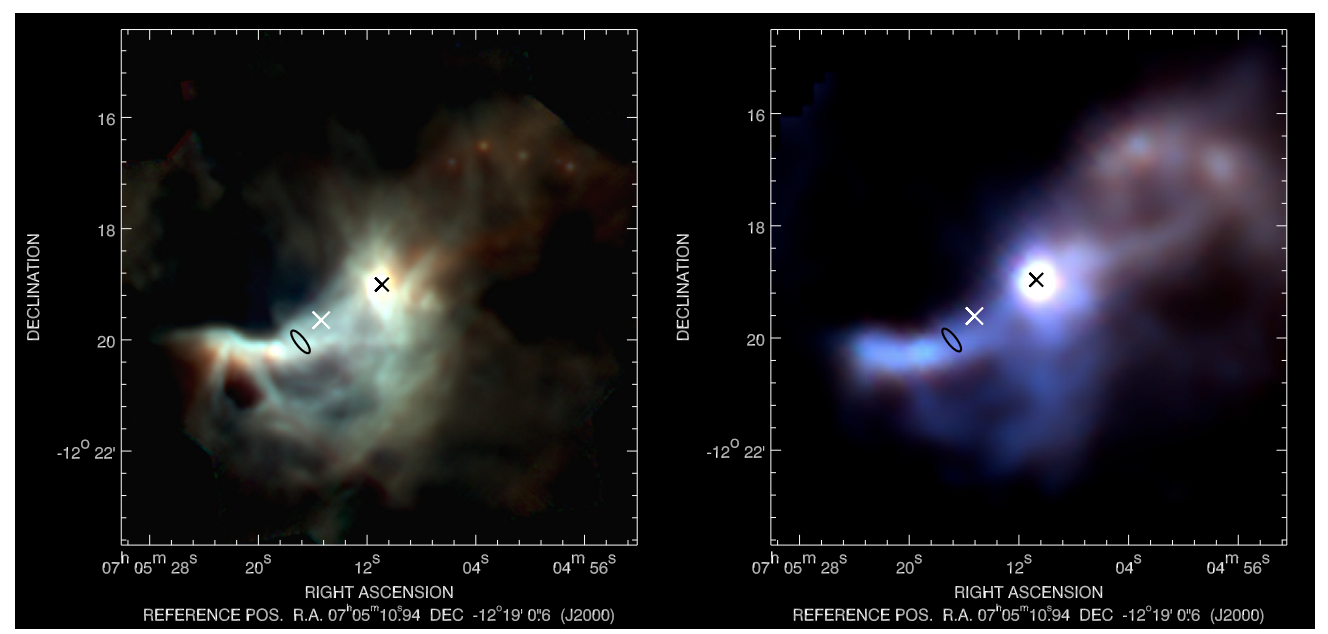

Fig. 1. Left: Star-forming complex around UYSO 1 with PACS. Three-colour composite with $70 \mu \mathrm{m}$ (blue), $100 \mu \mathrm{m}$ (green), and $160 \mu \mathrm{m}$ (red). Black cross at reference position: location of UYSO 1 derived by interferometry (see Fig. 2), white cross: peak of Sh 2-297 cm emission, ellipse: error ellipse of IRAS 07029-1215 position. Right: SPIRE three-colour composite with $250 \mu \mathrm{m}$ (blue), $350 \mu \mathrm{m}$ (green), and $500 \mu \mathrm{m}$ (red).

Table 1. Table of positions of relevant objects in the Herschel field.

\begin{tabular}{lll}
\hline \hline ID & $\begin{array}{l}\text { RA (J2000) } \\
{[\mathrm{h}: \mathrm{m}: \mathrm{s}]}\end{array}$ & $\begin{array}{l}\text { Dec (J2000) } \\
{\left[{ }^{\circ}:^{\prime}:^{\prime \prime}\right]}\end{array}$ \\
\hline IRAS 07029-1215 & $07: 05: 16.9$ & $-12: 20: 02$ \\
HD 53623 & $07: 05: 16.75$ & $-12: 19: 34.5$ \\
Sh-2 297 & $07: 05: 15.4$ & $-12: 19: 39$ \\
\hline UYSO 1 $^{a}$ & $07: 05: 11.14$ & $-12: 19: 00.2$ \\
LDN 1657A-1 $^{b}$ & $07: 04: 57.14$ & $-12: 16: 57.4$ \\
LDN 1657A-2 & $07: 04: 58.03$ & $-12: 16: 50.8$ \\
LDN 1657A-3 & \\
LDN 1657A-4 & $07: 05: 00.69$ & $-12: 16: 44.7$ \\
LDN 1657A-5 $^{b}$ & $07: 05: 03.50$ & $-12: 16: 33.7$ \\
\hline
\end{tabular}

Notes. ${ }^{(a)}$ derived from astrometry-adjusted PACS $70 \mu \mathrm{m}$ map (see Sect. 3.1); ${ }^{(b)}$ derived from MIPS $24 \mu \mathrm{m}$ map (see Sect. 3.2).

Table 2. Point source fluxes (without colour correction). The overall uncertainties are $20 \%$ for all wavelengths except for $160 \mu \mathrm{m}(25 \%)$.

\begin{tabular}{lrrrr}
\hline \hline ID & $\begin{array}{r}F_{v}[\mathrm{Jy}] \\
(24 \mu \mathrm{m})\end{array}$ & $\begin{array}{r}F_{v}[\mathrm{Jy}] \\
(70 \mu \mathrm{m})\end{array}$ & $\begin{array}{r}F_{v}[\mathrm{Jy}] \\
(100 \mu \mathrm{m})\end{array}$ & $\begin{array}{r}F_{v}[\mathrm{Jy}] \\
(160 \mu \mathrm{m})\end{array}$ \\
\hline UYSO 1 $^{a}$ & - & 168 & 239 & 193 \\
LDN 1657A-1 & 0.07 & 0.6 & 0.8 & 1.5 \\
LDN 1657A-2 & 0.10 & 0.2 & 0.2 & $<0.2$ \\
LDN 1657A-3 & 0.09 & 0.5 & 0.9 & 1.3 \\
LDN 1657A-4 & $<0.002$ & 0.3 & 1.1 & 2.6 \\
LDN 1657A-5 & 0.04 & 0.4 & 0.5 & 0.7 \\
\hline
\end{tabular}

Notes. ${ }^{(a)}$ Photometry obtained with large aperture (see Sect. 3.1). The corresponding SPIRE fluxes used for Fig. 3 are: $(91.1 \pm 13.7) \mathrm{Jy}$ $(250 \mu \mathrm{m}),(32.5 \pm 4.9) \mathrm{Jy}(350 \mu \mathrm{m})$, and $(11.2 \pm 1.7) \mathrm{Jy}(500 \mu \mathrm{m})$.

the detector sub-matrices were performed. Finally, the data were highpass-filtered, using a median window of the size of the full scan legs, to remove the effects of bolometer sensitivity drifts during data acquisition and 1/f noise. We masked out emission structures (visible in a first iteration) before computing and subtracting this running median; this step minimises oversubtraction of source emission. Finally, the data were projected onto a coordinate grid using the photProject routine inside HIPE, and flux calibration factors (Poglitsch et al. 2010) were applied.

Maps at 250, 350, and $500 \mu \mathrm{m}$ were obtained with SPIRE (Griffin et al. 2010) on October 19, 2009. Two 9' scan legs covered the source with the nominal speed of $30^{\prime \prime} / \mathrm{s}$. The data were

Page 2 of 4 processed within HIPE with the standard photometer script up to level 1. During baseline removal, we masked out the highemission area in the center. For these observations, no cross-scan data was acquired; therefore the resulting maps still show residual striping along the scan direction. An iterative destriping algorithm (Bendo et al. 2010) was applied to mitigate this effect. We adopt $F W H M$ beam sizes of $18^{\prime \prime} .1,25^{\prime \prime} .2$, and $36^{\prime \prime} 9$ at 250 , 350 , and $500 \mu \mathrm{m}$, respectively.

\section{Results}

The Herschel data show the detailed structure of the entire starforming complex containing UYSO1. The data are shown in Fig. 1, where we have combined the PACS and SPIRE data into two colour composites. In the southern region, there is a ridge of far-IR emission roughly oriented east-west. The extended emission there covers the formal position of IRAS 07029-1215. The IRAS positions, however, have usually been derived from the IRAS $12 \mu \mathrm{m}$ maps. We note that the reported position coincides with neither the much stronger far-IR peak associated with UYSO 1 (see below) nor the peak position of centimetre emission arising from the HII region (cf. Fig. 1). Parts of this emission ridge represent a PDR adjoining the HII region. Nevertheless, the bright condensation east of the IRAS position within this PDR ridge might correspond to another embedded compact core.

While this southern ridge is the brightest source at mid-IR wavelengths $(\lambda \lesssim 25 \mu \mathrm{m})$, the Herschel data present a far different picture. The emission area around UYSO 1 dominates the Herschel bands: UYSO 1 itself is situated on top of a highly structured emission plateau (not detected in the SCUBA $450 \mu \mathrm{m}$ data of Forbrich et al. 2004) with a similar extent and structure as the $850 \mu \mathrm{m}$ map by Forbrich et al. (2004). The emission in the close eastern vicinity $\left(\sim 30^{\prime \prime}\right)$ of UYSO 1 is also a PDR excited by the B star as indicated by the [O I] line observed by Forbrich et al. (2009). We do not delve into a detailed study of the temperature distribution for the whole region (see Stutz et al. 2010, for CB 244). However, from inspection of Fig. 1 it is apparent that the extended emission around UYSO1 has its maximum shortwards of $160 \mu \mathrm{m}$, while the region extending to the north-west contains emission that only becomes pronounced at the SPIRE wavelengths, indicating that even colder material is located in the LDN 1657A dark cloud. 


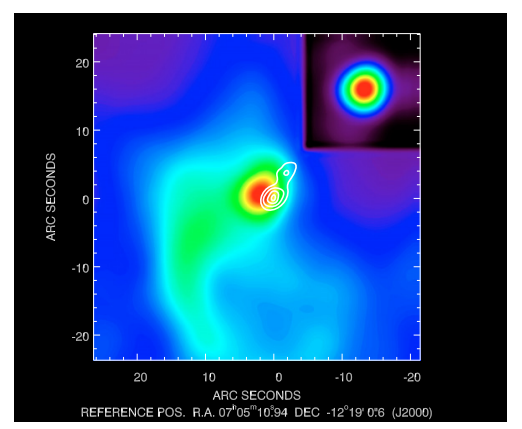

Fig. 2. PACS $70 \mu \mathrm{m}$ image of the central region of UYSO 1. The inset shows the PACS $70 \mu \mathrm{m}$ PSF (Poglitsch et al. 2010), rotated and scaled to match the UYSO 1 observation. The white contours denote the SMA $349 \mathrm{GHz}$ continuum detection (H. Beuther, priv. comm.)

\subsection{UYSO 1}

At far-IR wavelengths, the UYSO 1 core dominates the continuum emission of the region. The PACS instrument offers unprecedented spatial resolution of around 5.' 6 for the $70 \mu \mathrm{m}$ filter providing the opportunity to relate the far-IR emission to highresolution data at other wavelengths. Forbrich et al. (2009) reported Spitzer/MIPS observations of UYSO 1 at $70 \mu \mathrm{m}$ as well as $3 \mathrm{~mm}$ interferometric data pinpointing the two continuum peaks in UYSO 1. A large position shift of $>9^{\prime \prime}$ was found between the $\mathrm{mm}$ interferometric sources and the $70 \mu \mathrm{m}$ peak. Thus, the authors concluded that the optical depth at the column density peak is still too high for a direct detection of the sources at $70 \mu \mathrm{m}$. This large discrepancy in peak positions is not confirmed by our PACS data. Figure 2 shows the PACS view on the central region around UYSO 1 . As a position reference for the two related $\mathrm{mm}$ sources, we use new SMA $349 \mathrm{GHz}$ continuum data (H. Beuther, priv. comm.) that are in very good positional agreement with the $3 \mathrm{~mm}$ PdBI data from Forbrich et al. (2009). The typical 1- $\sigma$ absolute pointing accuracy of Herschel is 2"; however, deviations of up to $4^{\prime \prime}$ have been reported for a few operational days. Fortunately, the PACS data reveal several point sources northwest of UYSO 1 (not covered by the MIPS $70 \mu \mathrm{m}$ data) that were also detected in the MIPS $24 \mu \mathrm{m}$ map (see Sect. 3.2). We concatenated the PACS astrometry to the MIPS $24 \mu \mathrm{m}$ astrometry (see Fig. 2). As a result, the $70 \mu \mathrm{m}$ peak is now close to the stronger of the two interferometric point sources. The two positions still do not perfectly coincide, but the remaining offset of $\sim 2^{\prime \prime}$ is of the order of our final pointing uncertainties. Thus, there is a clear close association between the $70 \mu \mathrm{m}$ peak and the millimetre source(s). As a test, we smoothed our PACS $70 \mu \mathrm{m}$ data to the MIPS $70 \mu \mathrm{m}$ spatial resolution of $21^{\prime \prime}$ by convolving with an appropriate beam and regridding the resultant map to the same pixel scale. The final map is similar to the MIPS data, and the new peak position is indeed offset from that in the original PACS data by over $10^{\prime \prime}$. Because of the almost 4 times coarser spatial resolution of Spitzer at $70 \mu \mathrm{m}$, the true peak position had been smoothed out and merged with the surrounding PDR emission, and thus appeared shifted.

The comparison with the PACS $70 \mu \mathrm{m}$ point-spread function (PSF) in Fig. 2 illustrates that UYSO 1 is not an unresolved point source. The PACS PSF has a characteristic triangular outer shape. However, UYSO 1 is marginally resolved at $70 \mu \mathrm{m}$; the slight elongation is along the connecting line between the two $\mathrm{mm}$ continuum sources. On the other hand, the stronger of the two outflows is also oriented in this north-west/south-east direction (cf. Forbrich et al. 2009, their Fig. 2). Therefore, both source multiplicity and warm material along the outflow cone can explain our findings.
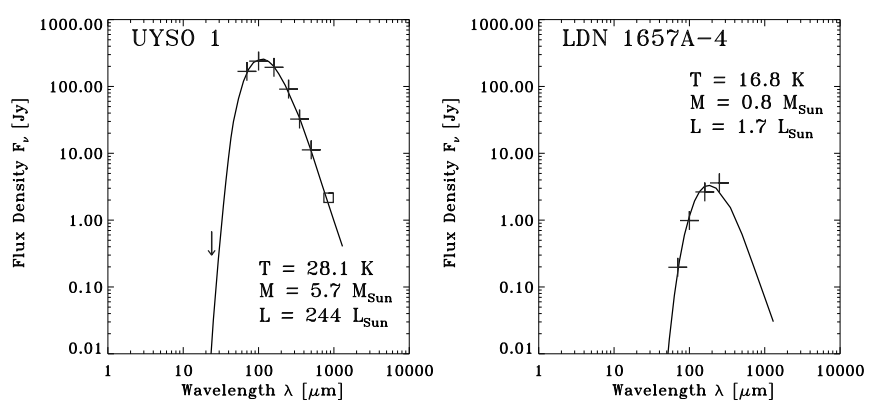

Fig. 3. Single-temperature modified black-body SED fits left for UYSO 1, and right for LDN 1657A-4. Plotted on the same scale, this illustrates the different natures of the two regions. Both results were obtained by adopting the Ossenkopf \& Henning (1994) opacities (their Table $1, \kappa$-column 5 ). For $T \lesssim 20 \mathrm{~K}$, colour correction terms for PACS fluxes become larger than the measurement uncertainties (mainly at $70 \mu \mathrm{m})$. They were applied to the LDN 1657A-4 fluxes before the fitting. Plus signs: Herschel data, square: SCUBA (Forbrich et al. 2004).

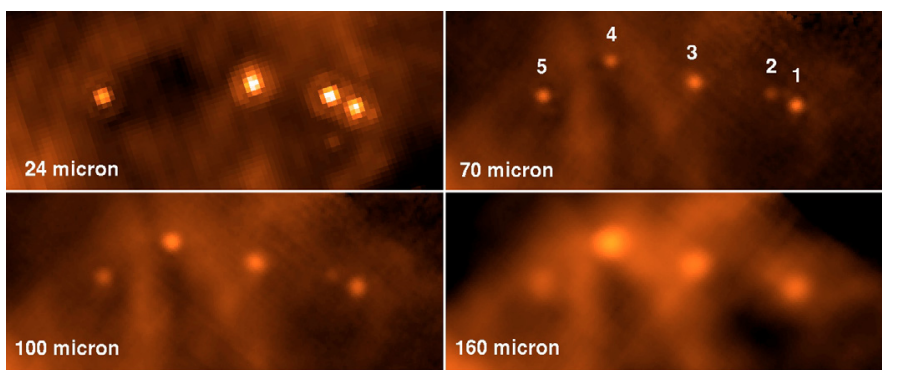

Fig. 4. Compact sources in the LDN 1657A region Table 1), north-west of UYSO 1 . The cutouts show a region of $220^{\prime \prime} \times 90^{\prime \prime}$ size at $24 \mu \mathrm{m}$ (upper left), $70 \mu \mathrm{m}$ (upper right), $100 \mu \mathrm{m}$ (lower left), and $160 \mu \mathrm{m}$ (lower right). Note especially the steep SED rise of source 4, spanning from a non-detection at $24 \mu \mathrm{m}$ to being the strongest source at $160 \mu \mathrm{m}$.

The SED of UYSO 1 was estimated by fitting a Planck function, modified by wavelength-dependent opacities, to the Herschel data. Our approach was to use fluxes derived from uniformly sized apertures. We chose a circle with an area equivalent to the SPIRE $500 \mu \mathrm{m}$ beam area $\left(1543^{\prime \prime 2}\right)$. We used the opacities of coagulated grains with thin ice mantles (Ossenkopf \& Henning 1994). One temperature component was sufficient to obtain a very good fit (see Fig. 3). The derived temperature of $\sim 28.1 \mathrm{~K}$ was lower than the previous estimate of $40 \mathrm{~K}$ in Forbrich et al. (2009), based on MIPS SED mode observations that probably covered more of the adjacent warmer PDR material. Nevertheless, our photometry also includes a small contribution from the PDR, which is heated from the outside, thus, the intrinsic luminosity is slightly overestimated (in principal agreement with simulations performed by Steinacker et al. 2006). Thanks to the clarified astrometry, actual Herschel fluxes for $\lambda<450 \mu \mathrm{m}$ could be included that exceed the Spitzer upper limits (which had been obtained after subtracting an extendedemission component) of Forbrich et al. (2009). Consequently, the derived luminosity $\left(244 L_{\odot}\right)$ is higher than their estimate $\left(50 L_{\odot}\right)$. We note that the derived total mass of $5.7 M_{\odot}$ strongly depends on the chosen dust model. We repeated the fitting with Draine \& Lee (1984) opacities, deriving instead a total mass of $26.4 M_{\odot}($ and $T=26.1 \mathrm{~K})$.

\subsection{New very cold source detections}

While UYSO 1 was the prime target of the Herschel observations, we also discovered new sources $3^{\prime}-4^{\prime}$ to the north-west of UYSO 1, within the neighbouring dark cloud LDN 1657A. 
From the SCUBA $850 \mu \mathrm{m}$ map shown in Forbrich et al. (2004), it was obvious that this region is associated with submillimetre emission. The PACS data resolve this region into a "pearl necklace" of five compact sources (Table 1). Nothing is known about these objects except the association of LDN 1657A3 with faint 2 MASS K band emission. There is no counterpart at $1.4 \mathrm{GHz}$ in the NVSS VLA survey. Unfortunately, the region is at the very edge of previous ROSAT X-ray observations (Gregorio-Hetem et al. 2009) and thus not properly covered. However, inspection of the entire dataset of MIPS $24 \mu \mathrm{m}$ data $^{1}$, of which just the central part was published in Forbrich et al. (2009), reveals that 4 of these 5 new sources are indeed detected (cf. Fig. 4). At wavelengths $\leq 160 \mu \mathrm{m}$, all these sources are unresolved. Only at SPIRE wavelengths does a more pronounced extended emission component become apparent (Fig. 1). Faint far-IR filaments seem to connect UYSO 1 and these northwestern sources. Hence, we assume that they are associated with the general star-formation complex. The distances between neighbouring LDN 1657A sources are quite similar $\left(39^{\prime \prime}-43^{\prime \prime}\right)$, only the two western sources 1 and 2 being closer together $\left(15^{\prime \prime}\right)$. At a distance of $1 \mathrm{kpc}$, this translates into around $0.2 \mathrm{pc}$ (and $0.073 \mathrm{pc}$, respectively) of projected distance. The distance $0.2 \mathrm{pc}$ is roughly the Jeans length of a cold (16-18 K), medium-dense $\left(1-2 \times 10^{4} \mathrm{~cm}^{-3}\right)$ gas. Thus, these sources most likely represent distinct cores. We report the PACS photometry in Table 2, based on PSF photometry (see also Henning et al. 2010) using the Starfinder tool (Diolaiti et al. 2000) and reference PSFs provided by the PACS instrument team. Most of these sources are detected at $24 \mu \mathrm{m}$, which is indicative of a second, warmer dust component.

Source LDN 1657A-4 has the steepest rising SED slope of all these sources (Figs. 3, 4). At $24 \mu \mathrm{m}$, the point source was undetected; we instead found a $24 \mu \mathrm{m}$ shadow at its location, caused by the high column density of the obscuring material associated with the cold core. We used the approach of Vasyunina et al. (2009) to transform the $24 \mu \mathrm{m}$ shadow contrast into a column density $N(\mathrm{H})$. The intensity ratio of the surrounding extended emission to the darkest pixel in the $24 \mu \mathrm{m}$ shadow was 1.51, after subtraction of the estimated zodiacal foreground of 25.56 MJy/sr. The optical depth was then $\tau=\ln 1.51$, and the column density was given by $N(\mathrm{H})=\tau / \sigma$. We assumed the extinction cross-section $\sigma$ from the $R_{\mathrm{V}}=5.5 \mathrm{~B}$ dust model ${ }^{2}$ of Weingartner \& Draine (2001), appropriate for the denser shielded regions of molecular clouds. We estimated a column density of $3.2 \times 10^{22} \mathrm{~cm}^{-2}$. This corresponds to $A_{\mathrm{V}}=26 \mathrm{mag}$, using the Ryter (1996) calibration. A more elaborate modelling of the fore- and background contributions (e.g., Stutz et al. 2007) would probably increase the calculated column density, but is beyond the scope of this letter. In addition, the standard (coarse) pixel scale of 2 .' 45 in the MIPS $24 \mu \mathrm{m}$ data used as well as an increased level of striping in the data limit the shadow contrast. Thus, the derived $24 \mu \mathrm{m}$ shadow column density represents a lower limit. Using the SPIRE $250 \mu \mathrm{m}$ peak flux measured at the position of LDN 1657A-4 (7.23 Jy/ 18'. 1 beam), the values of Ossenkopf \& Henning (1994) opacities at this wavelength $\left(18 \mathrm{~cm}^{2} / \mathrm{g}\right)$, and the temperature derived from the SED fit to the photometry data ( $17 \mathrm{~K}$, see Fig. 3, right), we also calculate a column density of $3.2 \times 10^{22} \mathrm{~cm}^{-2}$. This value is certainly a lower limit, since the relatively large SPIRE beam smears out the true column density peak. Nevertheless, the very

\footnotetext{
1 The region has not yet been observed with Spitzer at wavelengths $<24 \mu \mathrm{m}$. The compact sources are not detected in the MSX images.

$21.29 \times 10^{-23} \mathrm{~cm}^{2} / \mathrm{H}$ for MIPS 24 isophotal wavelength of $23.68 \mu \mathrm{m}$.
}

good agreement between the two independent methods is compelling. Finally, integration of the $24 \mu \mathrm{m}$ extinction map over the SPIRE $250 \mu \mathrm{m}$ beam area results in a total mass of $1.6 M_{\odot}$, a higher value than that derived from SED fitting $\left(0.8 M_{\odot}\right)$ but still comparable.

\section{Conclusions}

We have presented new Herschel PACS and SPIRE scan map data for the star-forming complex containing the intermediatemass core UYSO 1. They show the dust emission structures associated with this region in unprecedented detail. The high spatial resolution of the PACS $70 \mu \mathrm{m}$ map facilitates the differentiation of the true emission peak position from emission arising from the surrounding PDR material. The PACS data show that the $70 \mu \mathrm{m}$ emission is closely associated with the known location of the central millimetre peak(s) in UYSO 1. This revises an earlier finding of large offsets (based on Spitzer/MIPS $70 \mu \mathrm{m}$ data). Hence, the measured Herschel photometry can be used with more confidence than for previous data to estimate the SED of UYSO 1. Since the core was not clearly detected at $24 \mu \mathrm{m}$ (which otherwise would indicate a distinct component of warmer dust), a one-temperature modified black-body component with $T=28 \mathrm{~K}$ fits the data between $70-500 \mu \mathrm{m}$ very well. While clearly not in the hot-core/hot-corino regime, this temperature is higher than the typical values of $<17-20 \mathrm{~K}$ found in other very young regions of star formation. The known outflow activity can be one explanation. However, the influence of the nearby PDR has also to be taken into account (Forbrich et al. 2009). The Herschel data reveal the serendipitous discovery of five compact very red sources, situated to the north-west of UYSO 1, within the neighbouring dark cloud LDN 1657A. These objects have lower temperatures than UYSO 1. A particularly intriguing object is LDN 1657A-4, which has a very red SED and exhibits a $24 \mu \mathrm{m}$ shadow. We note that two different methods for the column density estimation provided very consistent results. For both objects, the Ossenkopf \& Henning (1994) grain opacities seem more suitable than the use of ISM grains.

Acknowledgements. PACS has been developed by an institute consortium led by MPE (Germany) and including UVIE (Austria); KU Leuven, CSL, IMEC (Belgium); CEA, LAM (France); MPIA (Germany); INAFIFSI/OAA/OAP/OAT, LENS, SISSA (Italy); IAC (Spain). It has been supported by the funding agencies BMVIT (Austria), ESA-PRODEX (Belgium), CEA/CNES (France), DLR (Germany), ASI/INAF (Italy), and CICYT/MCYT (Spain). We thank the anonymous referee for a constructive report.

\section{References}

Bendo, G. J., Wilson, C. D., Pohlen, M., et al. 2010, A\&A, 518, L65 Diolaiti, E., Bendinelli, O., Bonaccini, D., et al. 2000, in AO Systems Technology, ed. P. L. Wizinowich, SPIE Conf. Ser., 4007, 879 Draine, B. T., \& Lee, H. M. 1984, ApJ, 285, 89

Forbrich, J., Schreyer, K., Posselt, B., et al. 2004, ApJ, 602, 843 Forbrich, J., Stanke, T., Klein, R., et al. 2009, A\&A, 493, 547 Gregorio-Hetem, J., Montmerle, T., Rodrigues, C. V., et al. 2009, A\&A, 506, 711 Griffin, M. J., Abergel, A., Abreu, A., et al. 2010, A\&A, 518, L3 Henning, Th., Linz, H., Krause, O., et al. 2010, A\&A, 518, L95 Ossenkopf, V., \& Henning, T. 1994, A\&A, 291, 943

Pilbratt, G. L., Riedinger, J. R., Passvogel, T., et al. 2010, A\&A, 518, L1 Poglitsch, A., Waelkens, C., Geis, N., et al. 2010, A\&A, 518, L2 Ryter, C. E. 1996, Ap\&SS, 236, 285

Steinacker, J., Bacmann, A., \& Henning, T. 2006, ApJ, 645, 920 Stutz, A. M., Bieging, J. H., Rieke, G. H., et al. 2007, ApJ, 665, 466 Stutz, A., Launhardt, R., Linz, H., et al. 2010, A\&A, 518, L87 Vasyunina, T., Linz, H., Henning, T., et al. 2009, A\&A, 499, 149 Weingartner, J. C., \& Draine, B. T. 2001, ApJ, 548, 296 